Steenson, T. I. \& Walker, N. (1957). J. gen. Microbiol. 16, 146-155

\title{
The Pathway of Breakdown of 2:4-Dichloro- and 4-Ghloro-2-methyl-phenoxyacetic Acid by Bacteria
}

\author{
BY T. I. STEENSON AND N. WALKER \\ Soil Microbiology Department, Rothamsted Experimental Station, \\ Harpenden, Hertfordshire
}

SUMMARY: The metabolism of 2:4-dichlorophenoxyacetic acid and 4-chloro-2methylphenoxyacetic acid by a strain of Flavobacterium peregrinum and an Achromobacter sp., respectively, has been studied. Bacteria from young cultures were more active than those from older ones in oxidizing these substrates. Evidence is presented that adapted organisms dissimilate 2:4-dichlorophenoxyacetic acid through 2:4-dichlorophenol and 4-chlorocatechol, and that 4-chloro-2-methylphenoxyacetic acid is dissimilated through 5-chloro-2-cresol. Bacteria grown on 2:4-dichlorophenoxyacetic acid do not oxidize any of the other five possible isomers, but can oxidize 2:4-dibromo-, 4-bromo-2-chloro-, 4-chloro-, and, to a small extent, 2-chlorophenoxyacetic acids.

That the selective herbicides, 2:4-dichlorophenoxyacetic acid (2:4-D) and 4-chloro-2-methyl-phenoxyacetic acids (MCPA) are subject to bacterial decomposition in the soil is now well known, and the fate of these substances in bacterial cultures has been investigated by a number of workers. Evans \& Smith (1954) suggested that 6-hydroxy-2:4-dichlorophenoxyacetic acid was an early oxidation product of 2:4-D in a culture of an unidentified bacterium. Audus (1952) used a soil-perfusion technique to study the kinetics of the decomposition of herbicides in fresh soil and obtained some evidence that 2:4-dichlorophenol was an intermediate in 2:4-D breakdown. He found that a soil enriched with organisms to decompose 2:4-D would also decompose MCPA and conversely, and so argued that the first step in decomposition was an attack on the part of the molecule common to both compounds, namely, the acetic acid residue, which he postulated was split off by hydrolysis to glycollic acid, leaving the corresponding phenol. However, in more recent work with liquid cultures of Bacterium globiforme Audus \& Symonds (1955) claimed to have detected at least two phytotoxic intermediates, differing from 2:4-D in $\boldsymbol{R}_{\boldsymbol{F}}$ values by paper chromatography, and so they suggested that the implications of their earlier cross-perfusion experiments would have to be reconsidered. Rogoff \& Reid (1956) isolated from soil a yellow Corynebacterium $\mathrm{sp}$. which could decompose up to $0 \cdot 3 \%$ 2:4-D in a mineral salts medium. 'This organism oxidized 3.8, 19 and 38 $\mu \mathrm{M}$ 2:4-D in Warburg manometers, but $38 \mu \mathrm{M}$ showed a toxic effect; the organism also oxidized 2:4-dichlorophenol. They detected in cultures the nearly quantitative conversion of the organicallybound chlorine in 2:4-D to the inorganic form and so concluded that the molecule suffered complete decomposition. Steenson \& Walker (1956), using cultures of two different herbicide-decomposing bacteria, showed that the bacterial oxidation of 2:4-D or MCPA depended on adaptive (induced) enzyme 
formation. No oxidation of either 6-hydroxy-2:4-dichlorophenoxyacetic acid or of 2:4-dichlorophenol at the concentration used was obtained, and it was concluded that neither compound was an intermediate in 2:4-D breakdown.

In the present work, the principle of simultaneous adaptation (Stanier, 1947) has been employed to study the metabolism of 2:4-D, MCPA and some related compounds by the two bacterial species which were described by Steenson \& Walker (1956). Organisms for manometric experiments were grown on mineral salts agar plates with 2:4-D or MCPA as carbon source and supplemented with a little yeast extract. Growth, however, was slow and it was necessary to incubate for 5, 6 or more days to obtain a moderate amount of organisms. When cultures were grown for not more than 3 days, even though the yield of organisms appeared small, these organisms were found to be much more active than older ones; this observation was the key to many of the results which follow.

\section{METHODS}

Medium. Agar plates were poured from a medium containing 2:4-D, 0.1 g.; $\left(\mathrm{NH}_{4}\right)_{2} \mathrm{HPO}_{4}, 0.05 \mathrm{~g}$; $\mathrm{KCl}, 0.02 \mathrm{~g}$.; $\mathrm{MgSO}_{4} .7 \mathrm{H}_{2} \mathrm{O}, 0.02 \mathrm{~g}$.; $\mathrm{FeSO}_{4} .7 \mathrm{H}_{2} \mathrm{O}$, $\mathbf{0 . 0 0 5} \mathrm{g}$.; Difco dehydrated yeast extract, $0.05 \mathrm{~g}$.; agar, $2.0 \mathrm{~g}$. in tap water, $100 \mathrm{ml}$. When necessary, MCPA was used in place of 2:4-D. All cultures were incubated at $25^{\circ}$.

Manometric methods. Bacteria were washed in $0.02 \mathrm{M}$-phosphate buffer ( $\mathrm{pH} \mathrm{6.98)} \mathrm{and} \mathrm{resuspended} \mathrm{in} \mathrm{the} \mathrm{same} \mathrm{buffer} \mathrm{solution.} \mathrm{The} \mathrm{nitrogen} \mathrm{content}$ of the suspension was determined by the usual micro-Kjeldahl procedure. The oxygen uptake by $1 \mathrm{ml}$. cell suspension (containing $0 \cdot 2-0.6 \mathrm{mg}$. total $\mathrm{N}$ ) was measured in Warburg manometers at $30^{\circ}$. The main cup of the Warburg vessel contained the suspension of organisms and enough phosphate buffer to give a final volume of $3 \mathrm{ml}$., the substrates being placed in the side bulb. The centre cup contained $\mathbf{0} \cdot 2 \mathrm{ml} . \mathbf{2 0} \%$ (w/v) aqueous potassium hydroxide.

Analytical methods. Chloride was determined by Mohr's method using 0.05 N-silver nitrate solution (Kolthoff \& Sandell, 1952). Phytotoxic effects (Audus \& Quastel, 1947) were detected by placing a 2-3 ml. sample in a Petri dish together with a piece of filter-paper and planting twenty mustard or cress seeds on the paper. The seeds used had a percentage germination of over $90 \%$.

Reference compounds. 3-Chloro-, 3:4-dichloro and 2:4-dibromo-phenoxyacetic acids were synthesized from the corresponding phenols by the method of Pokorny (1941). 2:3-, 2:5- and 3:5-dichlorophenoxyacetic acids were given to us by Professor R. L. Wain; 2:5- and 2:6-dichlorophenoxyacetic acids by Professor G. E. Blackman; 6-hydroxy-2:4-dichlorophenoxyacetic acid by Dr G. W. K. Cavill; 2-hydroxy-4-chlorophenoxyacetic acid and 3:5-dichlorocatechol by Messrs Monsanto Chemical Company; 4-chlorocatechol by Professor R. T. Williams. Other substances were obtained commercially. 


\section{RESULTS}

Effect of age on activity of adapted cells

The Achromobacter strain was grown on agar plates containing MCPA and after 3, 6 and 10 days of incubation, respectively, batches of organisms were harvested, washed in buffer solution and used for manometric experiments. The rates of oxygen uptake by organisms from cultures of different ages were
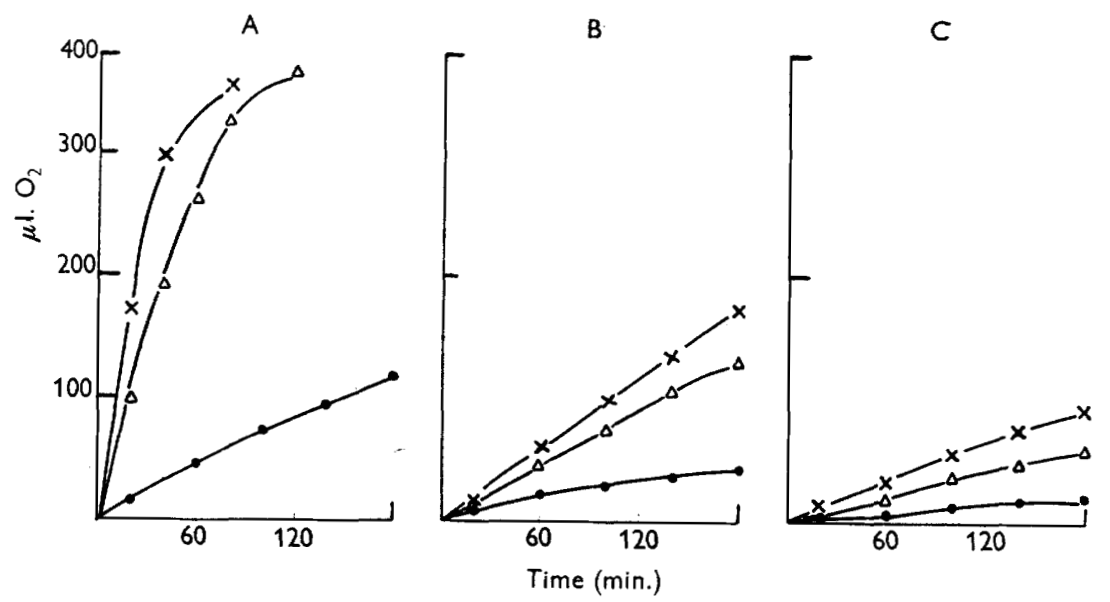

Fig. 1. Comparison of MCPA-grown Achromobacter organisms from cultures of different ages. Oxygen uptake by washed organisms $(-\rightarrow)$ alone and in the presence of $2 \mu$ mole MCPA $/ 3 \mathrm{ml} .(\Delta-\Delta)$ or $2 \mu$ mole $2: 4-\mathrm{D} / 3 \mathrm{ml} .(x-x)$. A, 3-day cultures; B, 6-day cultures; C, 10-day cultures.

determined in the presence of $2 \mu$ mole $/ 3 \mathrm{ml}$. of 2:4-D and MCPA as substrates. The results, plotted in Fig. 1, show that organisms from 3-day cultures completed the oxidation of the substrate in about $1 \frac{1}{2} \mathrm{hr}$. whereas less than $50 \%$ oxidation occurred, even after $4 \mathrm{hr}$., when using organisms from 6- or 10-day cultures. Similar results (Fig. 2) were obtained with Flavobacterium peregrinum, (Stapp \& Spicher, 1954) grown on 2:4-D-containing medium. The importance of using only young cultures in manometric experiments with these organisms was thus demonstrated.

\section{Oxygen uptake experiments with isomers of 2:4-D}

Washed organisms from 3-day cultures of the Achromobacter strain, grown on 2:4-D medium, were used to test the five isomeric compounds 2:3-, 2:5-, 2:6-, 3:4- and 3:5-dichlorophenoxyacetic acids as substrates in an experiment to measure rates of oxygen uptake. None of these compounds was oxidized by the 2:4-D-adapted bacteria.

In a similar experiment, but using 2:4-D-grown organisms of the Flavobacterium peregrinum strain, none of the isomers of 2:4-D was oxidized to any significant extent, although in some cases the rates of oxygen uptake were slightly above the endogenous rate (see Fig. 3). It was evident, therefore, that 
A

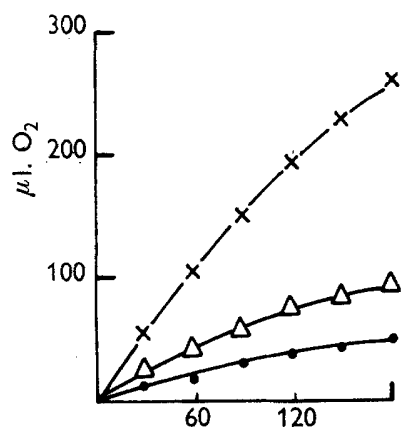

B

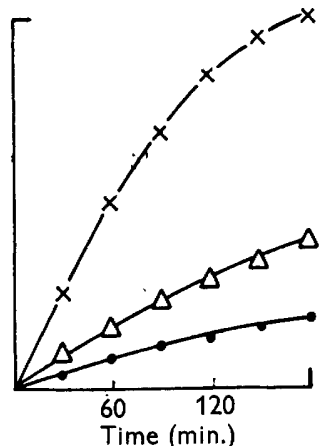

C

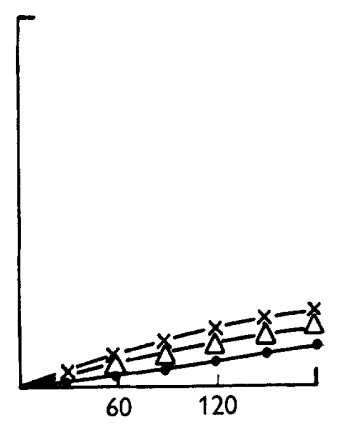

Fig. 2. Comparison of 2:4-D-grown Flavobacterium peregrinum organisms from cultures of different ages. Oxygen uptake by washed organisms alone $(-<)$ and in the presence of $2 \mu$ mole MCPA $/ 3 \mathrm{ml}$. $(\triangle-\triangle)$ or $2 \mu$ mole $2: 4-\mathrm{D} / 3 \mathrm{ml} .(\times-\times)$; A, from 3-day cultures; $B$, from 5-day cultures; $C$, from 9-day cultures.

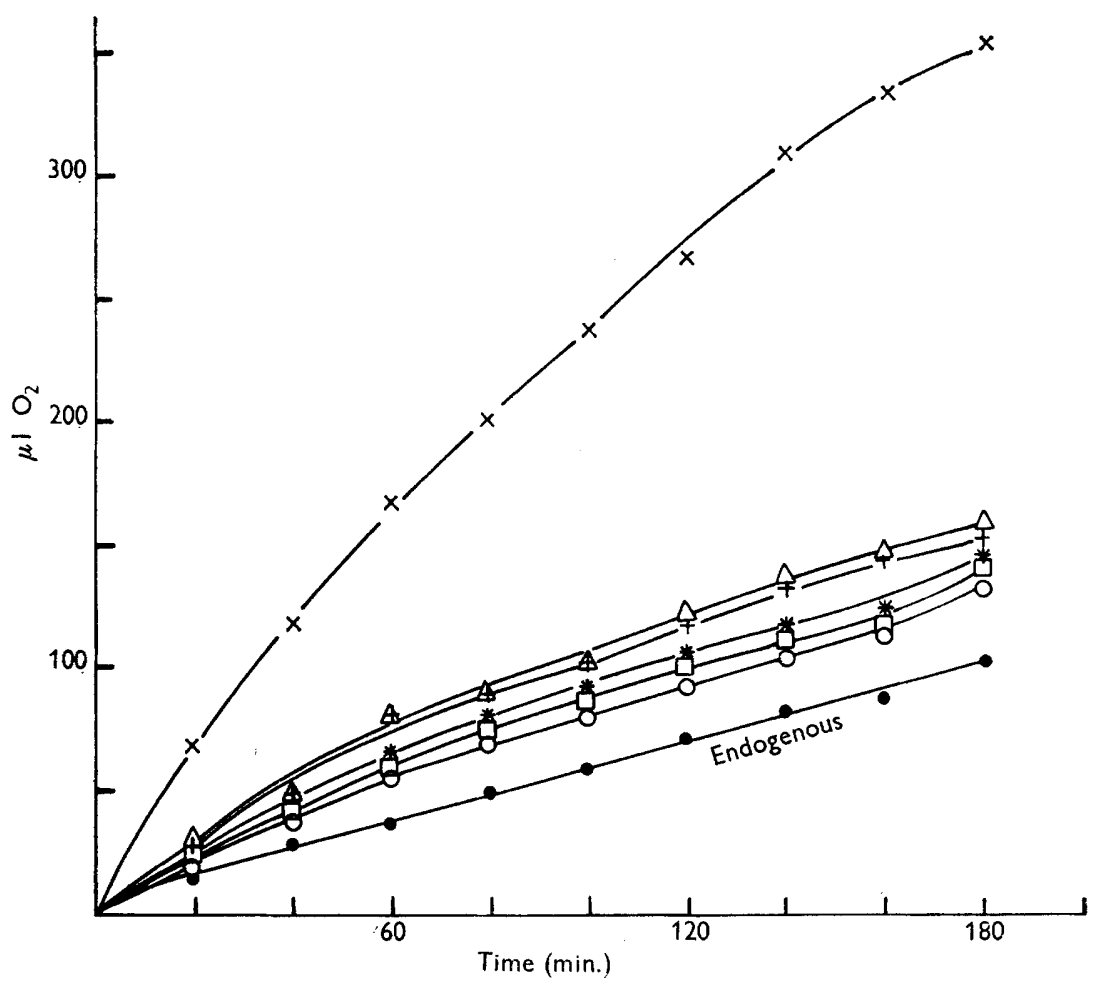

Fig. 3. Rate of oxygen uptake by washed, 2:4-D-grown Flavobacterium peregrinum organisms alone (-) and in the presence of $2 \mu \mathrm{mole} / 3 \mathrm{ml}$. 2:3-dichloro- $(\triangle-\triangle), 2: 4$-dichloro$(\times-\times)$, 2:5-dichloro- $(+-++)$, 2:6-dichloro- $(\mathrm{O}-\mathrm{O})$, 3:4-dichloro- (*and 3:5-dichlorophenoxyacetic acid $(\square-\square)$ as substrate. 
growth in the presence of $2: 4-\mathrm{D}$ resulted in organisms with a specific adaptation to metabolize 2:4-D and none of the isomers.

\section{Oxidation of mono-chlorophenoxyacetic acids}

Rates of oxygen uptake were measured for washed Achromobacter organisms which had been grown on 2:4-D medium, with 2-, 3- or 4-chlorophenoxyacetic acid as substrate. The results (Fig. 4) show that 4-chlorophenoxyacetic acid

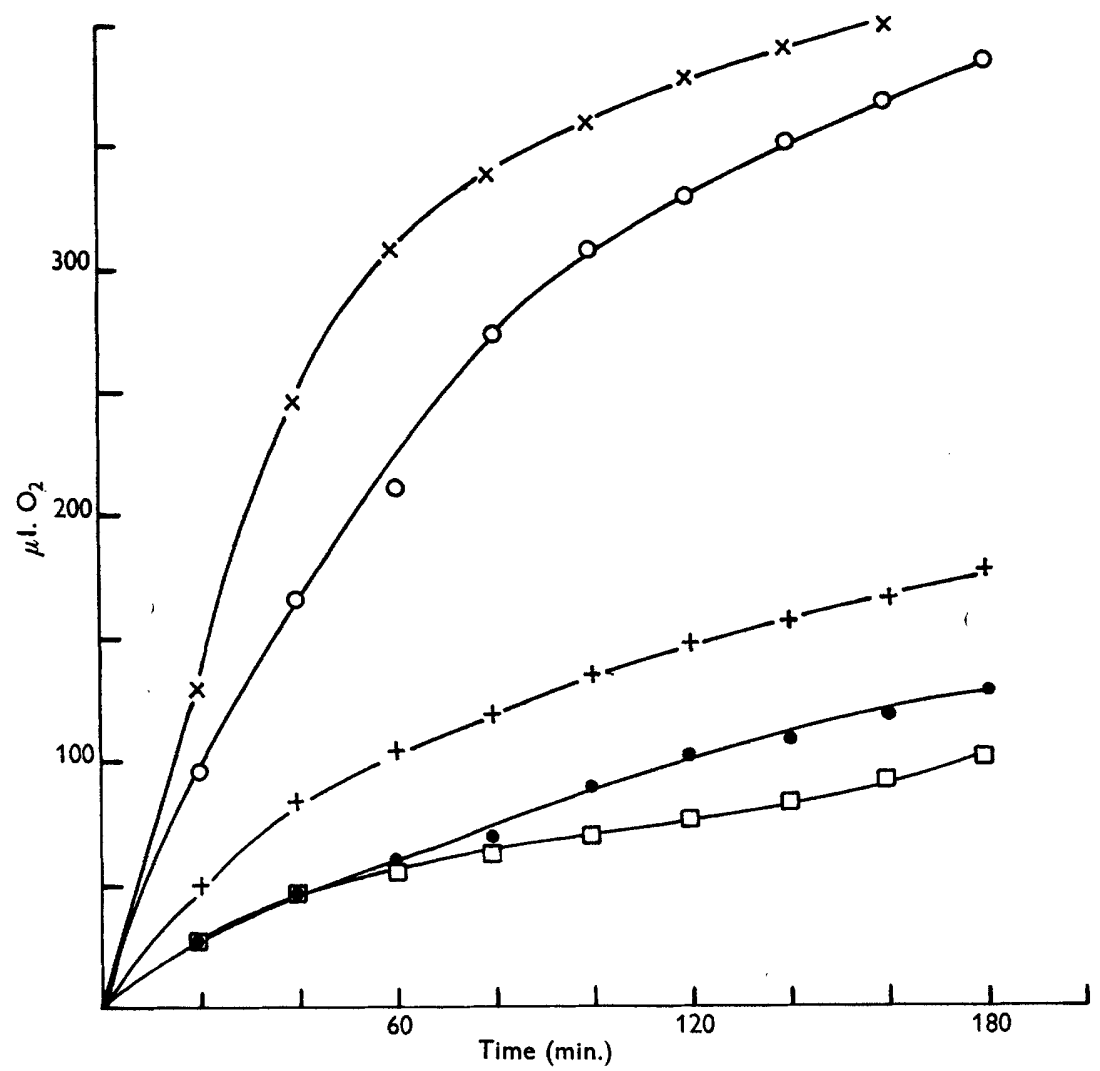

Fig. 4. Rate of oxygen uptake by Achromobacter organisms grown on 2:4-D. In presence of $2 \mu \mathrm{mole} / 3 \mathrm{ml}$. 2-chloro- $(+-+)$, 3-chloro- $(\square-\square)$, 4-chloro- $(O-O)$ and 2:4-dichlorophenoxyacetic acid $(\times-\times)$ as substrate.

was oxidized at nearly the same rate as 2:4-D; 2-chlorophenoxyacetic acid was oxidized at a slower rate and to a much lesser extent, but the 3-chloro-compound was not oxidized and even depressed slightly the endogenous respiration. Similar results were obtained with washed Flavobacterium peregrinum organisms which had been grown with 2:4-D.

\section{Cross-adaptation experiments}

Since, when grown on MCPA medium, the Achromobacter strain was adapted to oxidize both MCPA and 2:4-D, an experiment was made to determine whether 2:4-D-adapted organisms could also oxidize MCPA. A culture of 
the Achromobacter strain was grown on 2:4-D medium and then a series of three successive transfers on to fresh media after every 2 or 3 days was made; samples of organisms from each transfer were collected separately and washed. Rates of oxygen uptake by these organisms, with 2:4-D or MCPA as substrate, were determined in the usual manner. The results (Fig. 5) showed that after
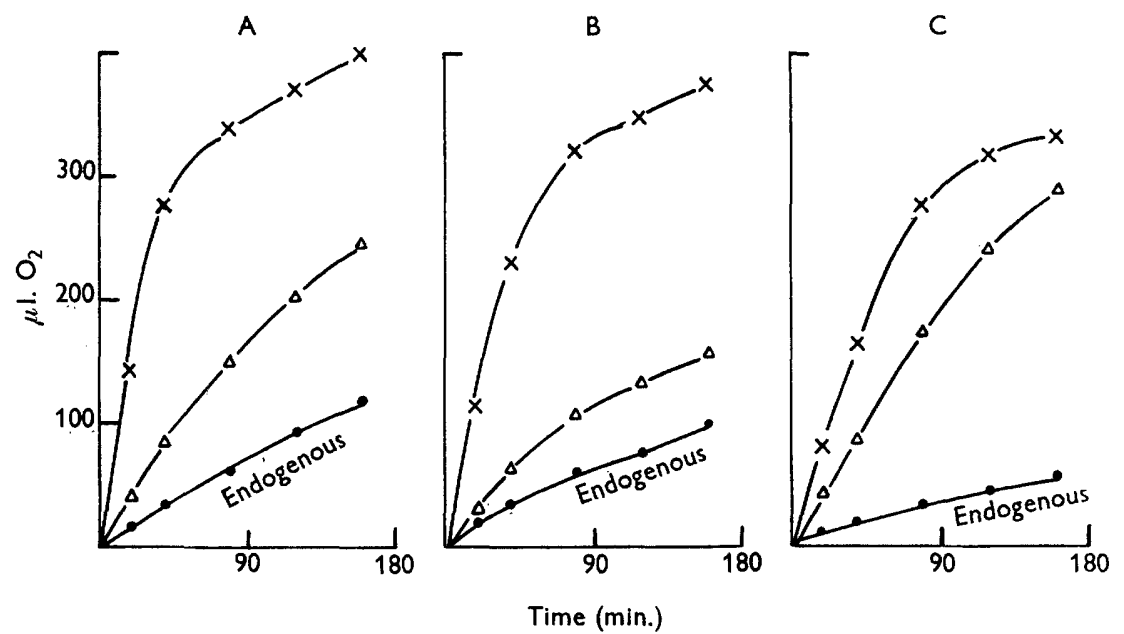

Fig. 5. Effect of successive transfers on 2:4-D medium on the rate of oxygen uptake by Achromobacter organisms compared with MCPA-grown organisms : in the presence of $2 \mu$ mole $/ 3$ ml. 2:4-D $(\times-\times)$ or MCPA $(\triangle-\triangle)$ as substrate. A, Organisms after one transfer on 2:4-D medium. B, Organisms after three transfers on 2:4-D medium. C, Organisms grown on MCPA after three previous subcultures on 2:4-D medium.

three transfers on 2:4-D medium, the organisms oxidized MCPA at a much slower rate than when first grown on 2:4-D; but when they were again grown on MCPA medium, the organisms recovered their former rate of MCPA oxidation.

\section{Oxidation of bromo-derivatives of phenoxyacetic acid}

The above results indicated that position isomerism was an important factor in determining the specificity of the adaptive enzymes of these organisms. It seemed of interest, therefore, to find whether substitution of chlorine by bromine would affect the susceptibility to oxidation of the substituted phenoxyacetic acid. Experiments showed that 2:4-D-grown organisms of either strain and MCPA-grown Achromobacter organisms all oxidized 2:4-dibromophenoxyacetic acid as fast as they did 2:4-D. 4-Bromo-2-chlorophenoxyacetic acid was oxidized similarly by 2:4-D-grown Achromobacter organisms.

2:4-Dibromophenoxyacetic acid could replace 2:4-D in media for the growth of either strain, so some experiments were carried out with Achromobacter organisms grown on the dibromo compound. The results obtained were similar to those given by 2:4-D-grown organisms (see Fig. 5B) in that the rate of oxygen uptake with 2:4-dibromo- or 2:4-dichlorophenoxyacetic acid was about the same, and with MCPA the rate of oxidation was much slower. 
Since it has been shown that 2:4-D- or MCPA-adapted bacteria oxidize 4-chloro-, 2:4-dichloro-, 4-chloro-2-methyl-, 4-bromo-2-chloro-, 2:4-dibromo-, and to some extent, 2-chlorophenoxyacetic acid, the conditions which determine the susceptibility of a mono- or di-substituted phenoxyacetic acid to oxidation may be summarized as follows: the first substituent should be a halogen and should occupy position 4 or 2 ; the second substituent in a 4-substituted compound should be in position 2 and may be either halogen or a methyl group.

\section{Oxidation of possible intermediate metabolites}

In earlier work, Steenson \& Walker (1956) failed to demonstrate the oxidation of 6-hydroxy-2:4-dichlorophenoxyacetic acid or of 2:4-dichlorophenol by 2:4-D-adapted bacteria; but since finding that organisms from young cultures were much more active in respiring on 2:4-D, it became important to reexamine the effect of such organisms on these compounds. The effect of substrate concentration, especially of antiseptic phenols, may also be critical; e.g. phenol is catabolized by certain bacteria at concentrations below $0.01 \mathrm{M}$, but above this it acts as a general antiseptic. Dichlorophenol is a stronger antiseptic than phenol, so it was necessary to examine the effect of this compound at low concentrations.

6-Hydroxy-2:4-dichlorophenoxyacetic acid. In experiments with washed organisms of either strain, grown on 2:4-D medium for not more than 3 days, no oxygen uptake was observed with 6-hydroxy-2:4-dichlorophenoxyacetic acid as substrate. With the Achromobacter strain, grown on MCPA medium for 3 days, there was no oxygen uptake, but the endogenous respiration was slightly depressed by 6-hydroxy-2:4-dichlorophenoxyacetic acid. Thus no evidence that the latter compound might be a metabolite of $2: 4-D$ was obtained.

2:4-Dichlorophenol. Fig. 6 shows the results of an experiment on the oxidation of 2:4-dichlorophenol by 2:4-D-grown Achromobacter organisms. One $\mu$ mole 2:4-dichlorophenol $/ 3 \mathrm{ml}$. was oxidized immediately at the same rate as 2:4-D, but with $2 \mu \mathrm{mole} / 3 \mathrm{ml}$. there was a moderate lag period before the rate of oxygen uptake reached that of 2:4-D. In another experiment it was found that $1.5 \mu$ mole 2:4-dichlorophenol $/ 3 \mathrm{ml}$. was oxidized immediately, but with $2 \mu \mathrm{mole} / 3 \mathrm{ml}$. a lag period was again observed, indicating a toxic concentration. Peptone-grown Achromobacter organisms did not oxidize 2:4-dichlorophenol at a concentration of $1 \mu \mathrm{mole} / 3 \mathrm{ml}$. The toxic effect of 2:4-dichlorophenol was demonstrated also in an experiment in which the oxidation by adapted organisms of $2 \mu$ mole $2: 4-\mathrm{D} / 3 \mathrm{ml}$. and a mixture of $2 \mu$ mole $2: 4-\mathrm{D}+2 \mu$ mole 2:4-dichlorophenol/3 ml. was compared. The results (Fig. 7) showed that there was a lag period before the mixed substrates were oxidized.

On the basis of Stanier's simultaneous adaptation hypothesis, therefore, it would appear that 2:4-dichlorophenol is an intermediary metabolite in 2:4-D dissimilation, the early stages of which involve the loss by oxidation of the acetic acid residue. It was therefore of interest to determine whether the phenols, at suitable concentrations, corresponding to the other phenoxyacetic 
acid derivatives subject to bacterial decomposition were oxidized immediately. Achromobacter organisms grown on 2:4-D oxidized 2:4-dibromophenol at a concentration not exceeding $\mathrm{M} / 3000$ (M/1500 was toxic), and 4-chlorophenol at $\mathrm{M} / 3000$ ( $\mathrm{M} / \mathbf{1 5 0 0}$ was slightly toxic). Neither phenol nor 2-chlorophenol was oxidized. MCPA-grown Achromobacter organisms oxidized 5-chloro-2-cresol at $M / 6000$ and, with a slight lag, at $M / 3000 ; 2: 4$-dichlorophenol at $M / 1500$; 2:4-dibromophenol at $\mathrm{M} / \mathbf{3 0 0 0}$; but not 2- or 4-cresol. Peptone-grown Achromobacter organisms did not oxidize m/3000 5-chloro-2-cresol.

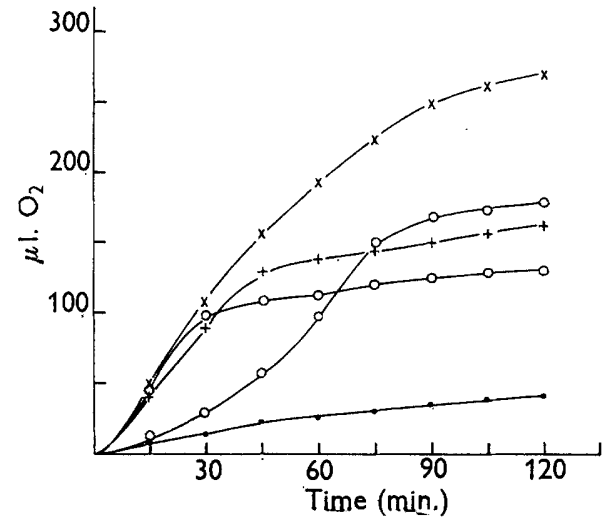

Fig. 6

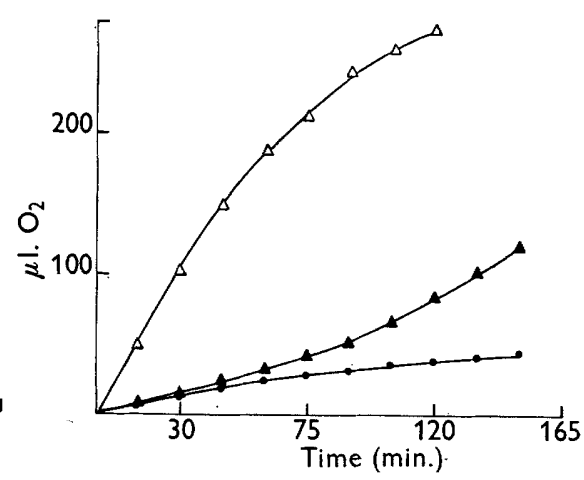

Fig. 7

Fig. 6. Rate of oxygen uptake by washed Achromobacter organisms (- $\longrightarrow$ ), grown on 2:4-D medium, and in the presence of $2 \mu$ mole 2:4-D/3 ml. $(x-\times), 2 \mu$ mole 4-chlorocatechol $/ 3 \mathrm{ml}$. $(+-+)$ and 1 and $2 \mu$ mole 2:4-dichlorophenol $/ 3 \mathrm{ml}$. $(\mathrm{O}-\mathrm{O})$.

Fig. 7. Effect of 2:4-D-grown Achromobacter organisms on 2:4-D in the presence of 2:4-dichlorophenol. Oxygen uptake by washed organisms alone $(-)$ and in the presence of $2 \mu$ mole $2: 4-\mathrm{D} / 3 \mathrm{ml} .(\triangle-\triangle)$ and a mixture $(\Delta-\Delta)$ of 2:4-D $(2 \mu \mathrm{mole})$ and 2:4-dichlorophenol ( $2 \mu \mathrm{mole})$.

4-Chlorocatechol. Washed Achromobacter organisms, grown on 2:4-D or MCPA media, oxidized 4-chlorocatechol immediately at the same rate as 2:4-D (Fig. 6). No oxygen uptake was observed with 2-hydroxy-4-chlorophenoxyacetic acid or 3:5-dichlorocatechol, but catechol was oxidized by 2:4-D-adapted organisms. Neither catechol nor 4-chlorocatechol was oxidized by peptone-grown organisms. After mixing the adapted organisms with catechol or 4-chlorocatechol, a deep yellow colour developed fairly quickly. The colour was discharged by acidifying with dilute aqueous hydrochloric acid and on shaking with ether, separating the ethereal layer and then extracting the latter with aqueous sodium hydrogen carbonate, the yellow coloured material was recovered. The yellow material showed similar behaviour therefore to the yellow acidic substance detected by us (Steenson \& Walker, 1956) in cultures of either strain growing in a liquid medium containing 4-chlorophenoxyacetic acid. The colour formed in the Warburg vessels was more intense than that in liquid cultures of 4-chlorophenoxyacetic acid medium. In similar manometric experiments with MCPA-grown organisms and 4-chlorophenol as substrate, a slow development of a pale yellow colour was observed, 
but it was much less intense than with 4-chlorocatechol. It seems possible that 4-chlorophenol may be oxidized through 4-chlorocatechol which subsequently gives rise to the yellow substance.

\section{DISCUSSION}

It is desirable to use as many criteria as possible in deciding whether a given compound is an intermediate in the bacterial dissimilation of an initial substrate; e.g. the detection of the supposed intermediate in cultures, conformity with Stanier's simultaneous adaptation hypothesis and the ability of the intermediate to support growth of the organism. Audus \& Symonds (1955) commented on the difficulties of growing adapted Bacterium globiforme in mineral salts + 2:4-D solution but they studied the growth rates of the organism and followed the rate of loss of phytotoxicity in cultures. They attempted to isolate intermediates by ether extraction of acidified cultures, but did not identify them. Our results from manometric experiments with adapted organisms of two bacterial species suggest the following scheme for the early stages in the metabolism of 2:4-D and MCPA:

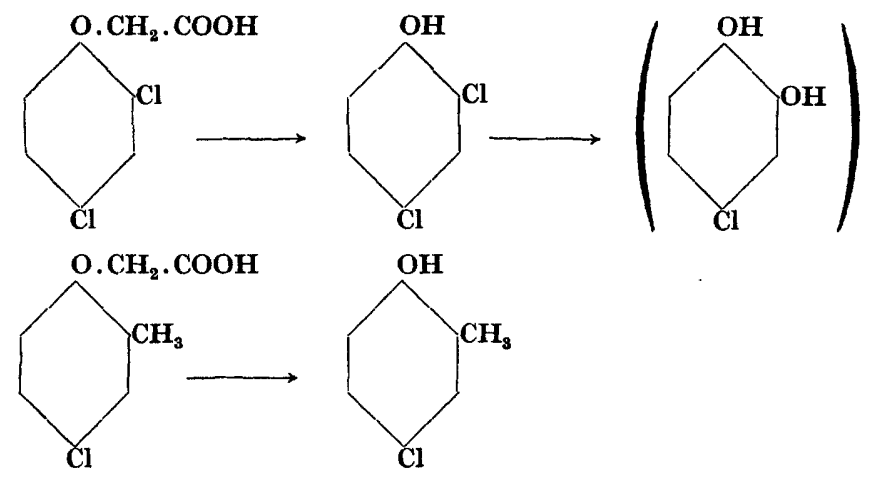

The first step seems to be the oxidation of the acetic acid moiety of the substituted phenoxyacetic acid to give the corresponding phenol, viz. 2:4-dichlorophenol and 5-chloro-2-cresol respectively. It is likely that 2:4-dichlorophenol is converted next to 4-chlorocatechol, although the evidence is rendered less decisive by the fact that adapted organisms can oxidize either catechol or 4-chlorocatechol. The failure to oxidize 3:5-dichlorocatechol is further support for the rejection of 6-hydroxy-2:4-dichlorophenoxyacetic acid as an intermediate. More evidence is required, however, to establish the precise reactions by which the chlorine atom is lost from the dichlorophenol.

The above pathway is not analogous to that which operates with 4-chlorophenoxyacetic acid, which Evans \& Smith (1954) found to be dissimilated through 4-chloro-2-hydroxyphenoxyacetic acid and 4-chlorocatechol. With 2:4-D metabolism, there is no evidence that 6-hydroxy-2:4-D is involved. It is interesting that when 2:4-D adapted organisms of our strains are inoculated into 4-chlorophenoxyacetate media, and incubated for 2 or 3 days, there is formed a yellow substance which appears to be identical with that formed by the action of suspensions of these organisms on 4-chlorocatechol or, to a smaller 
extent, on 4-chlorophenol. The same organisms do not oxidize 4-chloro-2hydroxyphenoxyacetic acid, so it seems likely that our 2:4-D-adapted organisms oxidize 4-chlorophenoxyacetic acid to 4-chlorocatechol via 4-chlorophenol and not through 4-chloro-2-hydroxyphenoxyacetic acid:

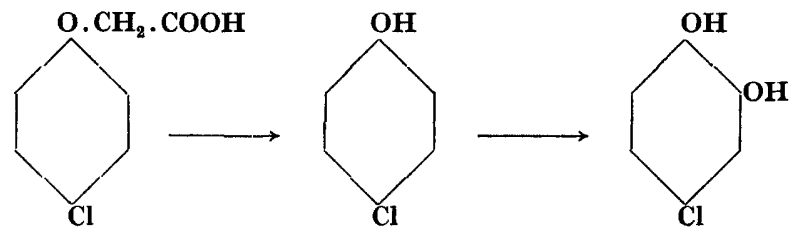

Whether the yellow substance is in fact 4-chloro-o-benzoquinone, or is derived from it, has not been established.

Rogoff \& Reid (1956) showed that 2:4-D-adapted organisms of a Corynebacterium sp. from soil could oxidize 2:4-dichlorophenol. Audus (1952) suggested that this compound might be an intermediate breakdown product of 2:4-D because he observed a rapid disappearance of 2:4-dichlorophenol when a dilute solution was perfused through soil crumbs previously enriched with 2:4-D. He noted also the toxic effect of an excess of 2:4-dichlorophenol. These findings are in agreement with our results obtained with pure cultures of bacteria and from the behaviour of washed bacteria in manometric experiments. Further study of the decomposition of 2:4-dichlorophenol and 5-chloro2-cresol by adapted bacteria is desirable.

We wish to thank Professors R. L. Wain, G. E. Blackman, R. T. Williams, Dr G. W. K. Cavill and Messrs Monsanto Chemical Company for gifts of chemicals, and Dr H. G. Thornton, For.Sec.R.S., for his interest and encouragement.

\section{REFERENCES}

Audus, L. J. (1952). The decomposition of 2:4-dichlorophenoxyacetic acid and 2-methyl-4-chlorophenoxyacetic acid in the soil. J. Sci. Fd Agric. 3, 268.

Audus, L. J. \& Quastel, J. H. (1947). Coumarin as a selective phytocidal agent. Nature, Lond. 159, 320.

Audus, L. J. \& Symonds, K. V. (1955). Further studies on the breakdown of 2:4-dichlorophenoxyacetic acid by a soil bacterium. Ann. appl. Biol. 42, 174.

Evans, W. C. \& Smrth, B. S. W. (1954). The photochemical inactivation and microbial metabolism of the chlorophenoxyacetic acid herbicides. Biochem.J. 57, xxx.

Kolthoff, I. M. \& SANDeld, E. B. (1952). Textbook of Quantitative Inorganic Analysis, 3rd ed. p. 451. New York: Macmillan Co.

Pokorny, R. (1941). Some chlorophenoxyacetic acids. J. Amer. chem. Soc. 63, 1768.

Rogoff, M. H. \& Reid, J. J. (1956). Bacterial decomposition of 2:4-dichlorophenoxyacetic acid. J. Bact. 71, 303.

Stanier, R. Y. (1947). Simultaneous adaptation. A new technique for the study of metabolic pathways. J. Bact. 54, 339.

STAPp, C. \& SpIcher, G. (1954). Untersuchungen über die wirkung von 2:4-D im boden. IV. Mitteilung: Flavobacterium peregrinum n.sp. und seine Fähigkeit zum abbau des hormones. Z. Bakt., II Abt. 108, 113.

Steenson, T. I. \& WAlker, N. (1956). Observations on the decomposition of chlorophenoxyacetic acids by soil bacteria. Plant \& Soil, 8, 17.

(Received 17 July 1956) 